\title{
Erratum to: Scalable temperature induced stress for the large-scale production of functionalized Bifidobacteria
}

\author{
Huu Thanh Nguyen ${ }^{1,2} \cdot$ Hary Razafindralambo ${ }^{3} \cdot$ Aurore Richel $^{4} \cdot$ Nicolas Jacquet $^{4}$. \\ Pol Evrard ${ }^{5}$ Patrice Antoine ${ }^{5} \cdot$ Philippe Thonart $^{2} \cdot$ Frank Delvigne $^{2}$
}

Published online: 5 March 2016

(C) Society for Industrial Microbiology and Biotechnology 2016

\section{Erratum to: J Ind Microbiol Biotechnol (2015)} 42:1225-1231

DOI 10.1007/s10295-015-1650-5

The addresses of the authors were given in French in the original article and some were mixed up. For a better visibility, the authors wish the addresses being published in English. These are the English written addresses in correct order.

The online version of the original article can be found under doi:10.1007/s10295-015-1650-5.

Huu Thanh Nguyen

nguyenhuuthanh@tdt.edu.vn

$\triangle$ Frank Delvigne

F.Delvigne@ulg.ac.be

1 Natural Products and Industrial Biochemistry Research Group, Faculty of Applied Sciences, Ton Duc Thang University, 19 Nguyen Huu Tho, Tan Phong, Ho Chi Minh, Vietnam

2 Microbial Processes and Interactions (MiPI), Agro-Bio Chem Department, University of Liège, Gembloux Agro-Bio Tech Faculty, Passage des Déportés 2, 5030 Gembloux, Belgium

3 Food Technology and Formulation, Agro-Bio Chem Department, University of Liège, Gembloux Agro-Bio Tech Faculty, Passage des Déportés 2, 5030 Gembloux, Belgium

4 Industrial Biological Chemistry, Agro-Bio Chem Department, University of Liège, Gembloux Agro-Bio Tech Faculty, Passage des Déportés 2, 5030 Gembloux, Belgium

5 THT Company s.a, Scientific Park of CREALYS, Camille Hubert 17, 5032 Gembloux, Belgium 